\title{
Design of compact joint transform correlator
}

\author{
Chao Fan
}

College of Information Science and Engineering, Henan University of Technology, Zhengzhou 450001, China

Received: 24.11 .2016

\begin{abstract}
To improve the quality of images taken with spatial cameras, we measure sub-pixel shifts between two adjacent images, using a joint transform correlator (JTC). First we describe the principles of measurements of the image shifts based on the JTC. Then, considering discrete pixel-like structures of a CCD and a spatial light modulator, we discuss relationships among the parameters of JTC components, issuing from the sampling theorem and the Fourier optics principles. Following from these points, we select the devices needed and design the Fourier lens to construct a compact JTC. Finally, we build the experimental platform and estimate the efficiency of measurements of the image shifts. The results show that sub-pixel image shifts can be measured very accurately, with the errors being less than 0.1 pixel under condition that the image shifts are within 1 pixel.
\end{abstract}

Keywords: joint transform correlator, image shifts, optical measurements, compact design

PACS: $42.15 . \mathrm{Eq}$

UDC: 535.8

\section{Introduction}

With increasing resolution of remote sensing cameras, image shifts become a major factor that affects the image quality. Because of complex working conditions associated with satellites, the image shifts are random and reveal almost no regularity. To decrease these shifts, the attitude of a satellite is required to be controlled in a very stable manner. This reduces the vibrations of the satellite but increases largely the cost and complexity of the apparatus. As a result, various image stabilization methods have been extensively studied. They can be divided into two kinds, a digital image method and an optical stabilization one $[1,2]$. No matter which of the methods is being used, the measurements of the image shifts represent a first step and a key procedure.

Being accompanied by the recent evolution of spatial light modulators (SLM) and CCD cameras, joint transform correlators (JTCs) have evolved from a purely optical system towards a hybrid computer-controlled photoelectric system. Now JTCs reveal a number of merits of the optical processing, e.g. fast parallel processing, large capacity and high speed, and can also be programmed conveniently. JTCs have been widely used in the image recognition and target detection [3-5]. In the present study, we use a JTC to measure the image shifts. Our idea is to input sampled adjacent sequential images into the JTC and compute the appropriate correlations basing on the distances between the actual cross-correlation peaks and the theoretical ones. The image shifts can be accurately obtained in this way.

The outline of this article is as follows. First the principles of measurements of the image shifts based on the JTC are given. Then, considering a discrete pixel-like structure of CCD and SLM, we discuss the relationships among the parameters of these devices, issuing from the Fourier optics fundamentals. Then a miniaturized JTC is designed and an experimental platform is built. Finally, we verify the measurement efficiency and the accuracy achieved for the image shifts. 


\section{The measurement principle of the image shifts based on JTC}

The structural scheme of an optoelectronic JTC suggested by us is displayed in Fig. 1. To make the structure of our JTC simple and compact, we employ the JTC constructed using a single Fourier lens (FL) and a single SLM. The reference image $r(x, y)$ and the target image $t(x, y)$ are loaded along an $x$ direction in a plane P1. Their centres are located at the points $\left(b_{r}, 0\right)$ and $\left(-b_{t}, 0\right)$. Compared with the reference image, the scene in the target image is moved some distances $\Delta x$ and $\Delta y$ along the $x$ and $y$ directions, respectively. Then the input of the JTC is given by

$$
E(x, y)=r\left(x-b_{r}, y\right)+t\left(x+\Delta x+b_{t}, y+\Delta y\right) .
$$

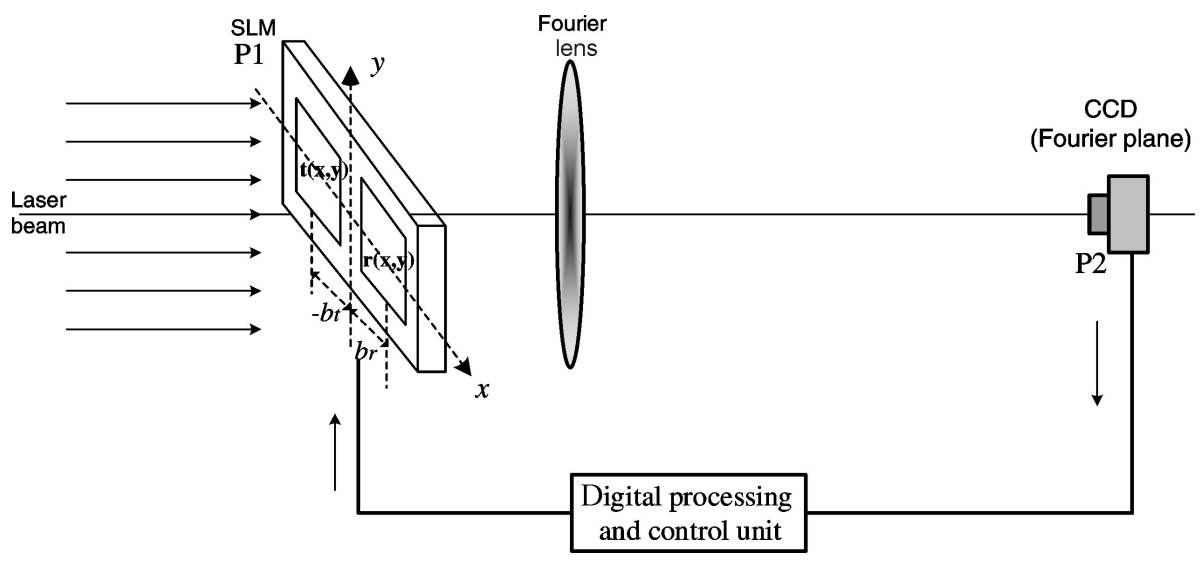

Fig. 1. Structural scheme of our optoelectronic JTC.

After transformation performed by the FL, one can calculate the joint spectrum $G(\xi, \eta)$ in the Fourier plane as

$$
G(\xi, \eta)=R(\xi, \eta) \exp \left[-2 i \pi b_{r} \xi\right]+T(\xi, \eta) \exp \left\{2 i \pi\left[\left(b_{t}+\Delta x\right) \xi+\eta \Delta y\right]\right\},
$$

where $R(\xi, \eta)$ and $T(\xi, \eta)$ are the Fourier transforms of $r(x, y)$ and $t(x, y)$, respectively. There is a CCD camera in the Fourier plane, which records the intensity distribution:

$$
\begin{gathered}
|G(\xi, \eta)|^{2}=|R(\xi, \eta)|^{2}+|T(\xi, \eta)|^{2}+R(\xi, \eta) T^{*}(\xi, \eta) \exp \left\{-2 i \pi\left[\left(b_{r}+b_{t}+\Delta x\right) \xi+\eta \Delta y\right]\right\}+ \\
T(\xi, \eta) R^{*}(\xi, \eta) \exp \left\{2 i \pi\left[\left(b_{r}+b_{t}+\Delta x\right) \xi+\eta \Delta y\right]\right\}
\end{gathered}
$$

where the symbol * implies complex conjugation. Controlled by a digital processing and control unit (see Fig. 1), the power spectrum is loaded to the SLM and transformed again by the FL. The complex amplitude distribution in the output plane of the system then reads as

$$
\begin{gathered}
C(x, y)=t(x, y) \otimes t(x, y)+r(x, y) \otimes r(x, y)+t(x, y) \otimes r(x, y) * \delta\left(x-b_{r}-b_{t}-\Delta x, y-\Delta y\right)+ \\
r(x, y) \otimes t(x, y) * \delta\left(x+b_{r}+b_{t}+\Delta x, y+\Delta y\right)
\end{gathered}
$$

where the symbols * and $\otimes$ now denote respectively convolution and correlation, and $\delta$ implies the Delta function. The first two items are auto-correlations and the last two cross-correlations. As evident from Eq. (4), when the reference image and the target image contain the same information, with a slight shifts $\Delta x$ and $\Delta y$, these shifts can be measured by finding the displacements between the actual cross-correlation peaks and the relevant ideal points $\left[ \pm\left(b_{r}+b_{t}\right), 0\right]$.

\section{Analysis of relationships among the parameter of JTC components}

Meanwhile, no construction parameters of the system components, such as the SLM, the lens and the CCD, have been considered. In any practical situation, both the SLM and the CCD contain 
discrete pixels that affect the image pattern according to the sampling theorem. Therefore we should study the relationships among the construction parameters of these components. Because the reference and target images are displayed along the horizontal direction on the SLM, we discuss only a single $x$ direction for simplicity. We suppose that, for the SLM shown in Fig. 2, the distance between the pixels is equal to $d_{1}$ and the width of the transparent zone is $w_{1}$. For the image loaded to the SLM, the sampling interval is given by the sampling theorem as $d_{1}$. Then the maximum spatial frequency which can be distinguished by the SLM reads as

$$
\xi_{\max }=\frac{1}{2 d_{1}}
$$

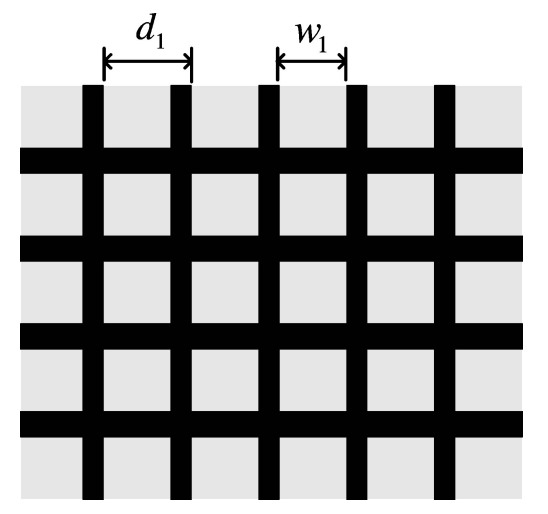

Fig.2 The pixel structure of SLM

The sampled input image $g\left(x_{1}\right)$ can be described in the plane P1 as [6]

$$
g\left(x_{1}\right)=\left\{\left[E\left(x_{1}\right) \sum_{m=-\infty}^{\infty} \delta\left(x_{1}-m d_{1}\right)\right] \operatorname{rect}\left(\frac{x_{1}}{w_{1}}\right)\right\} \times \operatorname{rect}\left(\frac{x_{1}}{L_{x}}\right),
$$

where $L_{x}$ is the total SLM width in the $x$ direction and the integer $m$ corresponds to the $m$-th order sampling. Supposing that there are $N_{1}$ pixels in the $x$ direction, we obtain $L_{x}=N_{1} d_{1}$. If the plane P1 is illuminated by a coherent plane-wave wave with the unit amplitude and a transformation is done by the $\mathrm{FL}$, we get the frequency function $G(\xi)$ in the output plane P2:

$$
G(\xi)=\left[E(\xi) * \frac{1}{d_{1}} \sum_{m=-\infty}^{\infty}\left(\xi-\frac{m}{d_{1}}\right)\right] w_{1} \operatorname{sinc}\left(w_{1} \xi\right) * L_{x} \operatorname{sinc}\left(L_{x} \xi\right),
$$

where $\xi=x_{2} /(\lambda f)$ and $x_{2}$ denotes the $x$ coordinate in the plane $\mathrm{P} 2$.

According to Eq. (7), the spectrum of the input image is periodic because of the discrete sampling by the SLM pixels, whereas the interval of the cycles is $(\lambda f) / d_{1}$ following from the diffraction principle. The width and the power distribution of the higher-order spectra are similar to those of the zero-order spectrum, with the difference that their amplitudes are modulated with the function $\operatorname{sinc}\left(w_{1} \xi\right)$. The latter induces a rapid decrease in the spectral powers with increasing order $m$. According to the diffraction spectrum shown in Fig. 3 and Eq. (7), the central spot represents a socalled diffraction point spread function of the Fourier transform system. Its width is $2 \lambda f /\left(N_{1} d_{1}\right)$, so that we have

$$
N_{2} d_{2} \geq \frac{\lambda f}{d_{1}},
$$

where $\mathrm{N}_{2} d_{2}$ is the CCD width along the $x$ direction. As a result, we obtain the inequality

$$
f \leq \frac{N_{2} d_{2} d_{1}}{\lambda} .
$$




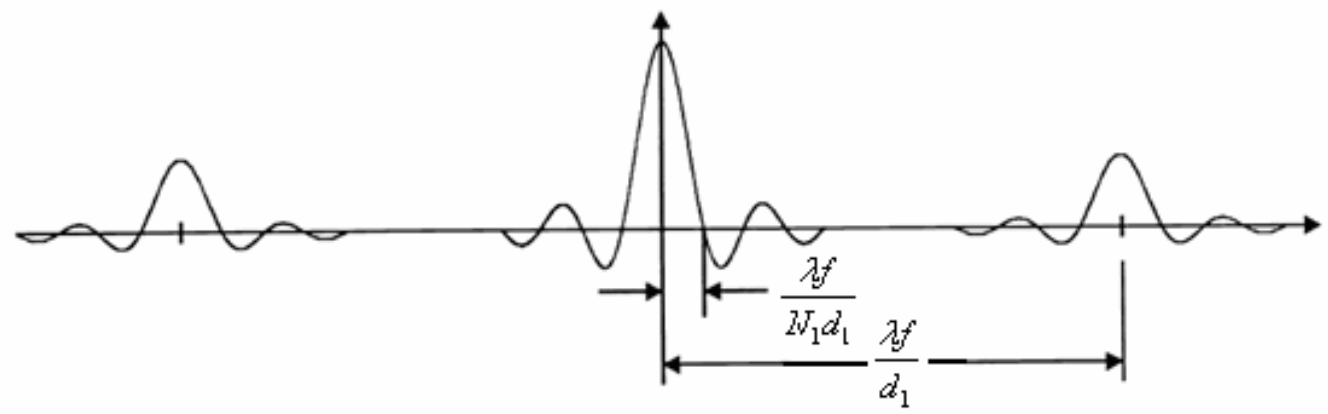

Fig. 3. Diffraction pattern caused by a discrete pixel-like structure of our SLM.

Usually, a joint power spectrum (JPS) can be better detected by the FL with a longer focal length. Hence, we select the focal length of the FL as

$$
f=\frac{N_{2} d_{2} d_{1}}{\lambda} .
$$

On the other hand, the JPS is formed by interference among the same points coming from the target and reference images. The spatial frequency of the fringe is $\left(b_{r}+b_{t}\right) /(\lambda f)$, according to the interference theory. When the JPS is detected with the CCD, the spatial resolution of the latter should be higher than that of the JPS, i.e. we have the condition

$$
\frac{b_{r}+b_{t}}{\lambda f} \leq \frac{1}{2 d_{2}}
$$

or

$$
b_{r}+b_{t} \leq \frac{\lambda f}{2 d_{2}}=\frac{N_{2} d_{1}}{2} .
$$

Eq. (12) describes the relationships among the distance between the target and reference images, the pixel size of the $\mathrm{CCD}$, the focal length of the FL, and the wavelength of the laser.

The JPS sampled by a pixel of the CCD can be described as follows:

$$
P(\xi)=\left\{\left[|G(\xi)|^{2} \sum_{m=-\infty}^{\infty} \delta\left(\xi-n \frac{d_{2}}{\lambda f}\right)\right] * \operatorname{rect}\left(\frac{\xi \lambda f}{d_{2}}\right)\right\} \times \operatorname{rect}\left(\frac{\xi \lambda f}{N_{2} d_{2}}\right) .
$$

Then, controlled by a computer, the JPS is re-input into the SLM. It can be written as

$$
P^{\prime}(\xi)=\left\{\left[\left|G\left(M \xi^{\prime}\right)\right|^{2} \sum_{m=-\infty}^{\infty} \delta\left(\xi^{\prime}-n \frac{d_{2}}{\lambda f}\right)\right] * \operatorname{rect}\left(\frac{\xi^{\prime} \lambda f}{d_{2}}\right)\right\} \times \operatorname{rect}\left(\frac{\xi^{\prime} \lambda f}{N_{2} d_{2}}\right) .
$$

Here $\xi$ is the spatial frequency coordinate of the JPS displayed at the SLM, $\xi=\xi / M$, $M=d_{2} / d_{1}$ is a scaling factor introduced by the difference in the pixel sizes of the CCD and the SLM, and $N_{2} d_{1}$ is the JPS width at the SLM. Because the $N_{2} d_{1}$ value is much larger than $w_{1}$, the diffraction effect can be ignored whenever the SLM is illuminated by a parallel laser beam. After Fourier transforming by the lens, the optical field distribution in the output plane is given by [7]

$$
C^{\prime}\left(x_{2}\right)=\frac{w_{2}}{d_{2}\left[C\left(x_{2}\right) \sum_{n=-\infty}^{\infty} \delta\left(x_{2}-\frac{n \lambda f}{d_{1}}\right)\right]} \times \operatorname{sinc}\left(\frac{w_{1} x_{1}}{\lambda f}\right) .
$$

Here 


$$
\begin{aligned}
C^{\prime}\left(x_{2}\right)= & \Im\left[\left|G\left(M \xi^{\prime}\right)\right|^{2}\right]=\left[r\left(\frac{x_{2}}{M}\right) \operatorname{rect}\left(\frac{x_{2}}{W M}\right)\right] \otimes\left[r\left(\frac{x_{2}}{M}\right) \operatorname{rect}\left(\frac{x_{2}}{W M}\right)\right] \\
& +\left[t\left(\frac{x_{2}}{M}\right) \operatorname{rect}\left(\frac{x_{2}}{W M}\right)\right] \otimes\left[t\left(\frac{x_{2}}{M}\right) \operatorname{rect}\left(\frac{x_{2}}{W M}\right)\right] \\
& +\left[r\left(\frac{x_{2}}{M}\right) \operatorname{rect}\left(\frac{x_{2}}{W M}\right)\right] \otimes\left[t\left(\frac{x_{2}}{M}\right) \operatorname{rect}\left(\frac{x_{2}}{W M}\right)\right] * \delta\left[x_{2}-M\left(b_{r}+b_{t}\right)\right] \\
& +\left[t\left(\frac{x_{2}}{M}\right) \operatorname{rect}\left(\frac{x_{2}}{W M}\right)\right] \otimes\left[r\left(\frac{x_{2}}{M}\right) \operatorname{rect}\left(\frac{x_{2}}{W M}\right)\right] * \delta\left[x_{2}-M\left(b_{r}+b_{t}\right)\right]
\end{aligned}
$$

where $W$ is the width of each input image. Note that the constant factor $1 / M^{4}$ in Eq. (16) is ignored.

It is seen from the above equations that, since the JPS is recorded by the CCD and is reloaded into the SLM again, the multi-level diffraction is induced in the output plane and the optical field is distributed periodically. In Fig. 4, a dashed line represents the modulation effect of the function $\operatorname{sinc}\left(w_{1} x_{2} / \lambda f\right)$ in Eq. (15), whose first zero points are located at $\pm \lambda f / w_{1}$, and the curve inside each dashed rectangle is determined by the function $C\left(x_{2}\right)$, which denotes the output of each level correlation. The centres of each level correlations are located at $(n \lambda f) / d_{1}$, where $n=0, \pm 1, \pm 2, \ldots$. The corresponding intensity decreases quickly with increasing $n$, and the distance between the two adjacent correlation peaks $\lambda f / d_{1}$ is determined by the pixel size of the SLM. Only a zero-level correlation peak is detected if one measures the image shifts by recording the locations of the correlated peaks.

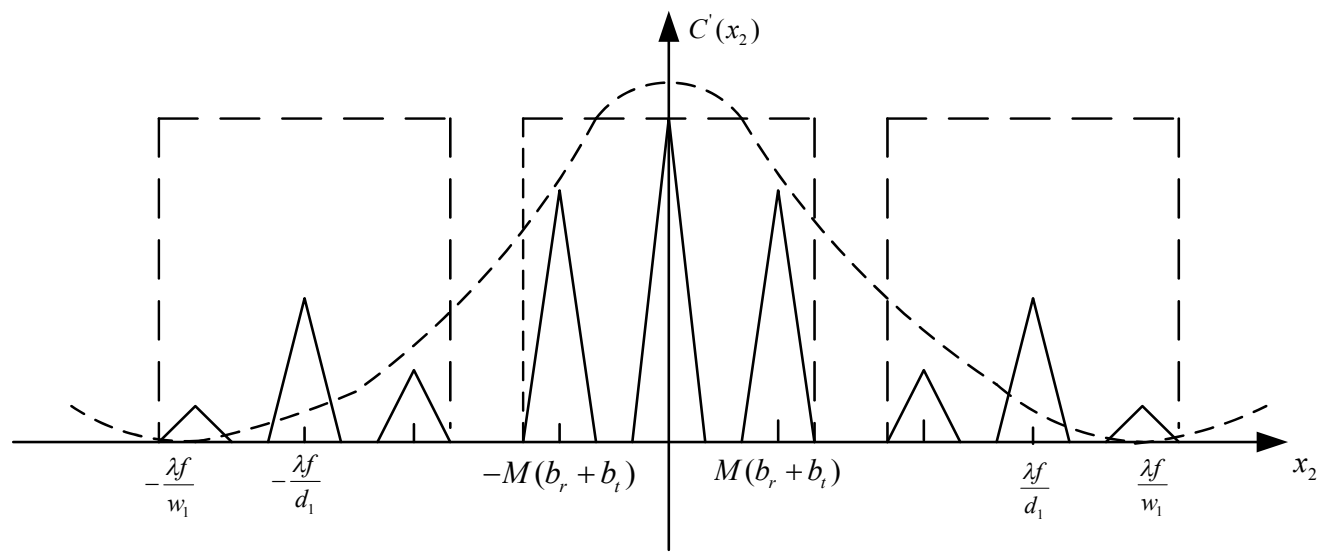

Fig. 4. Output spatial distribution of the correlation signal.

According to Eq. (16), there are four terms in the zero-level correlation. The first and the second ones are auto-correlation peaks, both of them being overlapped at the origin, with the width $2 M W$. The third and the fourth terms are cross-correlations related to the image shifts, which should be detected accurately. Their centres are located at $\pm M\left(b_{r}+b_{t}\right)$ and the width of each is also equal to $2 M W$. To avoid overlapping among the auto-correlations and cross-correlations, one has to meet the condition

$$
M\left(b_{r}+b_{t}\right) \geq 2 M W,
$$

or

$$
b_{r}+b_{t} \geq 2 W .
$$

The cross-correlations of the zero and first levels should also be avoided: 


$$
M\left(b_{r}+b_{t}\right)+M W \leq \frac{\lambda f}{2 d_{1}} .
$$

Since we have $M=d_{2} / d_{1}$, the above equation can be rewritten as

$$
b_{r}+b_{t}+W \leq \frac{\lambda f}{2 d_{2}} .
$$

Comparing Eqs. (12) and (20), one can see that the sum $\left(b_{r}+b_{t}\right)$ satisfying Eq. (20) is enough. Because the pixel sizes of the SLM and the CCD used in the JTC are restricted by the manufacturing technology, they cannot be selected at random. To satisfy the relationship deduced above, the other parameters (i.e., the focal length of the FL, the width of the input image, and the distance between the target and reference images) must be adjusted properly basing on the above equations.

\section{The design of miniaturized JTC}

\subsection{Selection of the components}

According to our design procedure, the SLM and the CCD are selected first of all since, as mentioned above, their parameters are restricted by the manufacturing technique. An electrically addressed device TFT-LCD XGA3 made by the Forth Dimension Displays Co. Ltd, England, has been used as an SLM. Its spatial resolution is $1024 \times 768$, the pixel pitch $18 \times 18 \mu \mathrm{m}^{2}$, the pixel dimensions $13 \times 10 \mu \mathrm{m}^{2}$, and the whole size $18.5 \times 13.9 \mathrm{~mm}^{2}$. We have used a device EL-400ME made by DTA Co. Ltd, Italy, as a CCD. Its resolution is $768 \times 512$, the pixel dimensions $9 \times 9 \mu \mathrm{m}^{2}$, and the total size $6.9 \times 4.6 \mathrm{~mm}^{2}$. A He-Ne laser with the wavelength $\lambda=0.6328 \mu \mathrm{m}$ has been used as a monochrome light interference source. Then the focal length of FL can be calculated with Eq. (10):

$$
f=\frac{N_{2} d_{2} d_{1}}{\lambda}=\frac{768 \times 9 \times 18}{0.6328}=196.6 \mathrm{~mm} .
$$

Since the SLM should be completely illuminated by a parallel laser beam, the aperture of the lens should be no less than the length of the SLM diagonal:

$$
D \geq \sqrt{18.5^{2}+13.9^{2}} \approx 23 \mathrm{~mm}
$$

where $D$ is the aperture of the FL.

\subsection{The design of Fourier lens}

To make the construction of JTC compact, we use a long-distance Fourier transform lens consisting of a positive lens and a negative lens (see Fig. 5). Since the above lenses are separated in the optical system, the main plane of the latter is shifted in the forward direction. Then the working distance is less than the focal length, which can efficiently shorten the total size of the FL.

Assume that the focal lengths of the lenses $L_{1}$ and $L_{2}$ are equal respectively to $f_{1}^{\prime}$ and $f_{2}^{\prime}$, the focal length of the $\mathrm{FL}$ is $f^{\prime}$, the distance between $L_{1}$ and $L_{2}$ is $t_{1}$, and the distance from $L_{2}$ to the CCD (i.e., to the focal plane) is $t_{2}$. Since, according to Eqs. (21) and (22), we have $f^{\prime}=196.6 \mathrm{~mm}$ and the aperture $D \geq 23 \mathrm{~mm}$, we let the latter parameter to be $D=25 \mathrm{~mm}$. Applying a simple geometrical optics, we obtain

$$
\begin{aligned}
& \frac{1}{t_{2}}-\frac{1}{f_{1}^{\prime}-t_{1}}=\frac{1}{f_{2}^{\prime}}, \\
& \frac{1}{f^{\prime}}=\frac{1}{f_{1}^{\prime}}+\frac{1}{f_{2}^{\prime}}-t_{1} \times \frac{1}{f_{1}^{\prime}} \times \frac{1}{f_{2}^{\prime}},
\end{aligned}
$$




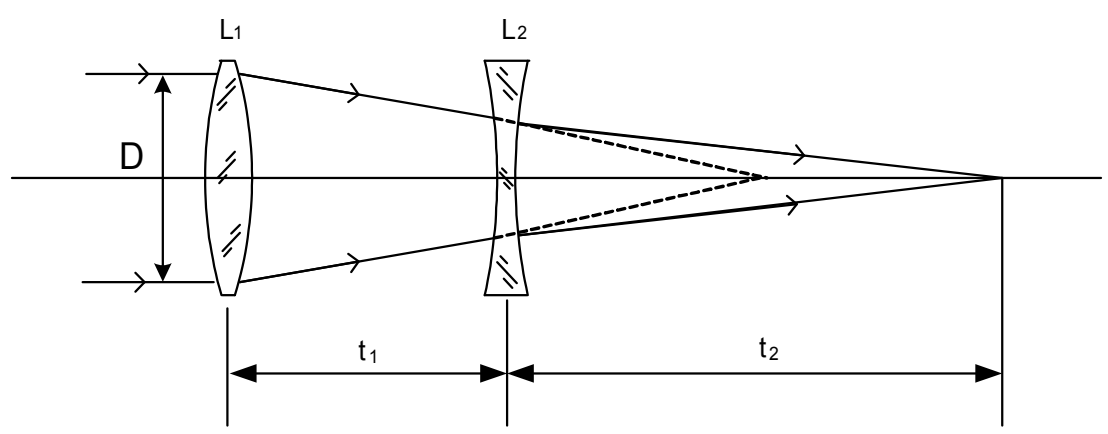

Fig. 5. Schematic diagram of a long-distance optical system.

$$
L=t_{1}+t_{2}
$$

Based on Eqs. (23) and (24), we express the parameter $t_{2}$ as

$$
t_{2}=\frac{f_{2}^{\prime}\left(f^{\prime}-t_{1}\right)}{f_{2}^{\prime}-t_{1}} .
$$

To make the JTC shorter, we substitute Eq. (26) into Eq. (25) and use the condition $\mathrm{d} L / \mathrm{d} t_{1}=0$ :

$$
\begin{aligned}
& t_{1}=t_{2}=t_{\min }=f_{2}^{\prime}\left[1-\sqrt{1-\frac{f^{\prime}}{f_{2}^{\prime}}}\right], \\
& f_{1}^{\prime}=\frac{f^{\prime}\left(t_{\min }-f_{2}^{\prime}\right)}{f^{\prime}-f_{2}^{\prime}} .
\end{aligned}
$$

Fig. 6 illustrates the relationships among the working distance $2 t_{1}$ and the focal lengths $f_{1}$ ' and $f_{2}^{\prime}$, which follow from the above equations for the particular case $f^{\prime}=196.6 \mathrm{~mm}$.

If we have $f_{2}^{\prime}=-40 \mathrm{~mm}$, Eqs. (27) and (28) result in $t_{1}=t_{2}=57.28 \mathrm{~mm}, f_{1}^{\prime}=80.84 \mathrm{~mm}$, $D_{1}=25 \mathrm{~mm}$ and $D_{2}=7.29 \mathrm{~mm}$. Hence, the relative apertures of $L_{1}$ and $L_{2}$ are equal to $1: 3.2$ and $1: 5.5$, respectively. If the focal length of $L_{1}$ is adjusted and $f_{1}{ }^{\prime}=100 \mathrm{~mm}$, the re-calculated working distances are $t_{1}=80.34 \mathrm{~mm}$ and $t_{2}=38.67 \mathrm{~mm}$. Then the total length of the FL becomes

$$
L=t_{1}+t_{2}=80.34+38.64=118.98 \mathrm{~mm} \text {. }
$$

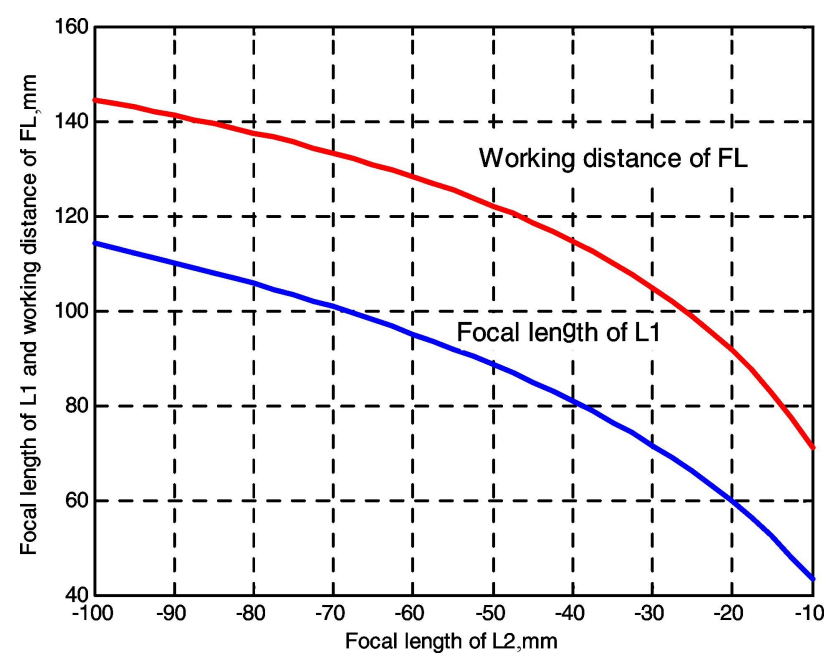

Fig. 6. Illustration of relationships among the working distance and the parameters $f_{1}^{\prime}$ and $f_{2}^{\prime}$. 
Finally, the aperture of the lens $L_{2}$ then reads as

$$
D_{2}=\frac{f_{1}^{\prime}-t_{1}}{f_{1}^{\prime}} \times D=\frac{100-80.34}{100} \times 25 \approx 5 \mathrm{~mm}
$$

After adjusting properly, the relative apertures become $D_{1} / f_{1}^{\prime}=1: 4$ and $D_{2} / f_{2}^{\prime}=1: 8$. Although the total length is now a little longer, the relative apertures of the lenses $L_{1}$ and $L_{2}$ are improved significantly, which is of benefit for the lens design. With the above parameters, the angle of the field of view of the FL is given by

$$
\omega=\arctan \left(\frac{y}{2 f^{\prime}}\right)=\arctan \left(\frac{\sqrt{6.9^{2}+4.6^{2}}}{2 \times 196.9}\right)=1.2^{\circ},
$$

where $y$ is the length of the CCD diagonal and $f^{\prime}$ the focal length of the FL. The resulting parameters of the long-distance FL calculated as explained above are listed in Table 1.

Table 1. Parameters calculated for our long-distance Fourier lens.

\begin{tabular}{cccc}
\hline Parameter & Fourier lens system & Lens $L_{1}$ & Lens $L_{2}$ \\
\hline Focal length, mm & 196.6 & 100 & -40 \\
Aperture, $\mathrm{mm}$ & 25 & 25 & 5 \\
Relative aperture & $1 / 8$ & $1 / 4$ & $1 / 8$ \\
Some positional & $t_{1}=80.34, t_{2}=38.64, L=118.98$ (total length) \\
parameters, mm & & $\omega=1.2^{\circ}$ \\
Field of view angle & &
\end{tabular}

Since we use a laser in our JTC, we have no need in considering chromatic aberration corrections. On the other hand, the spherical aberration and the sine condition are still essential because the optical system of the FL has a little field-of-view angle and a large aperture. Then a doublet can be used for the lens $L_{1}$, thus benefiting the aberration correlation. Similarly, a laser beam-expansion system can also use the identical long-distance lens. The final structure of the optical system of our compact JTC is shown in Fig. 7. The total length of the JTC is given by

$$
L_{J T C}=118.98+118.98+40=277.96 \approx 278 \mathrm{~mm} .
$$

Even though some extensions can be compromised, the total length is no longer than $300 \mathrm{~mm}$, which reduces efficiently the total size of the JTC.

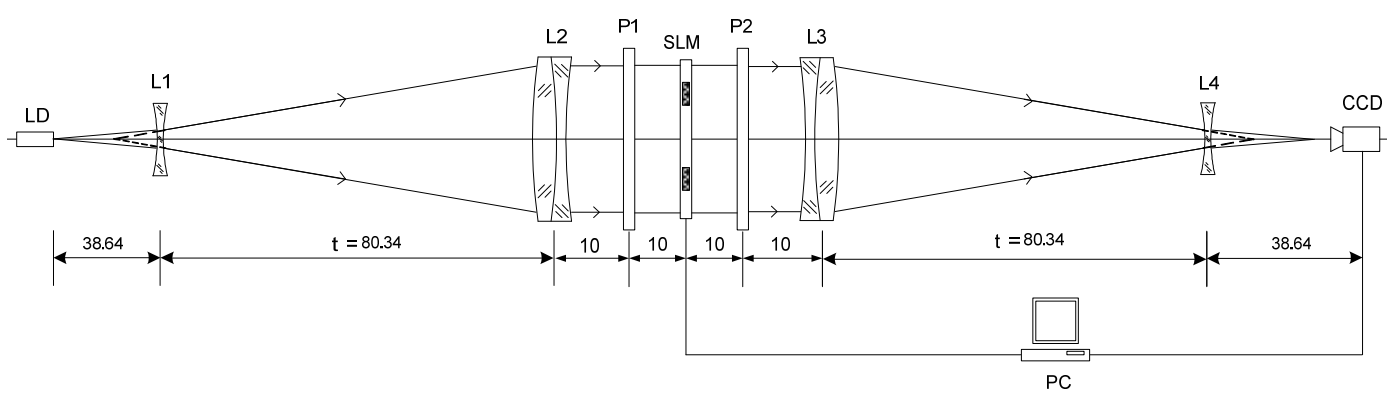

Fig. 7. General schematic diagram of our miniaturized JTC. 


\section{Image shift measurements based on the JTC}

Our experimental platform has been built using the components described above with the purpose of checking the effectiveness of the system and the measurement accuracy. In our experiment, an initial sub-image with the size of $256 \times 256$ is extracted from the remote sensing image, after being interpolated and down-sampled. The target image with the sub-pixel shifts and the reference image are then obtained, with the same sizes $128 \times 128$. Then the both images are input into the SLM. According to the relationships derived in Section 3, the distance between the images is 200 pixels (see Fig. 8a). The output associated with the correlation of these two images is shown in Fig. 8b. The auto-correlations and the two cross-correlations represent large disperse spots separated some distance from each other. This is similar to the pattern shown in the central slashed rectangle in Fig. 4. It is evident that the measurement error is larger than 1 pixel if the image shift is directly measured from the output.

For improving the measurement accuracy one has to weaken the auto-correlation power, which makes the cross-correlations shrink into little bright points. There are many processing methods for this aim, which allow finding the appropriate centroids easily [8-11]. After having done a lot of experiments, we have found that the measurement accuracy becomes the highest when the power spectra of the target and reference images are first subtracted from the JPS. Then the subtracted power spectrum is binarized with a zero threshold, as shown in Fig. 8c. A lot of strips are contained in the power spectrum, which means that the JPS represents an interference pattern formed by numerous identical points coming from the target and reference images. Their intensities are modulated by the diffraction of the target image (or the reference image). After being subtracted, the interference strips that contain the information on the image shifts are only remained in the JPS. The processed JPS is again Fourier transformed by the FL and the correlation output is caught by the CCD, as shown in Fig. 8d. Now we find that the cross-correlations shrink into two little spots with more distinct edges and higher powers, if compared with Fig. 8b. This allows for determining the centroids of the cross-correlations peaks easily. As a consequence, the image shifts can be measured with notable higher accuracy.

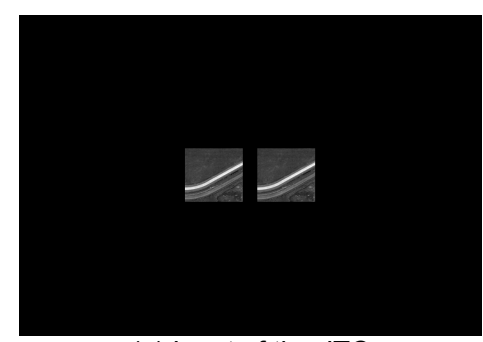

(a) Input of the JTC

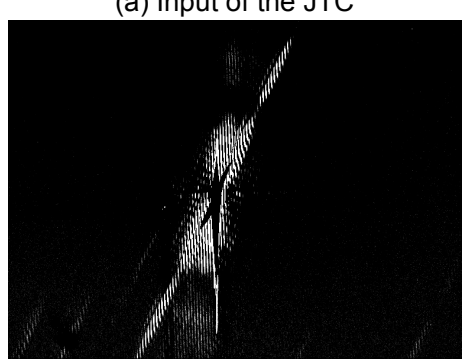

(c) Subtracted and binarized JPS
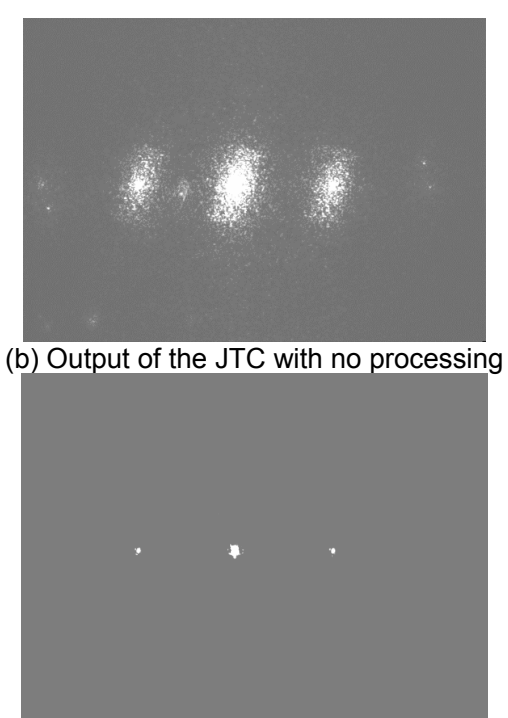

(d) Output of the JTC after processing

Fig. 8. Illustrations of inputs and outputs of our JTC. 
Basing on our experimental platform, we have studied the measurement performances of the JTC on the examples of 100 different sub-images, whose sizes are $256 \times 256$. After that, the initial and shifted images have been interpolated and merged with software to form the target image and the reference image with the same sizes $128 \times 128$. The image shift between them is less than 1 pixel. We have input these sub-images into our JTC and the image shift has been measured. The results analyzed using standard mathematical statistics have demonstrated that the image shifts can indeed be measured very accurately and the mean measurement error for all of the images remains less than 0.1 pixel.

\section{Conclusion}

To improve the quality of images taken with high-resolution spatial camera, we have suggested using a specific JTC for measuring the shifts between the two adjacent images. Considering the miniaturization requirements, we have analyzed the relationships among the parameters of the components of our optical system, basing on the Fourier optics principles. After having completed these stages, we have designed a compact JTC and built a corresponding experiment platform. By employing certain processing methods associated with our JPS, we have been in a position to detect easily the locations of the cross-correlation points and so measure accurately the relevant image shifts. The results obtained have shown that the measurement errors do not exceed 0.1 pixel, whenever the shifts between the target and reference images are less than 1 pixel.

\section{Acknowledgments}

This work is supported by the Science and Technology Support Project of the State Grain Administration (No 201413001), the China Scholarship Council (No 201408410255) and the Natural Science Foundation of Henan Province (No 162300410062).

\section{References}

1. Chiu C W, Chan P C and Wu D Y, 2007. Optimal design of magnetically actuated optical image stabilizer mechanism for cameras in mobile phones via genetic algorithm. IEEE Trans. Magn. 43: 2582-2584.

2. Hyun Choi, Jong-Pil Kim, Myeong-Gyu Song, Wan-Chin Kim, No-Cheol Park, Young-Pil Park, and Kyoung-Su Park, 2008. Effects of motion of an imaging system and optical image stabilizer on the modulation transfer function. Opt. Express. 16: 21132-21141.

3. Thomas B, Miffre A, David G, Cariou J P, and Rairoux P, 2012. Remote sensing of trace gases with optical correlation spectroscopy and lidar: Theoretical and numerical approach. Appl. Phys. B. 108: 689-702.

4. Thomas B, David G, Anselmo C, Cariou J-P, Miffre A, and Rairoux P, 2013. Remote sensing of atmospheric gases with optical correlation spectroscopy and lidar: First experimental results on water vapor profile measurements. Appl. Phys. B. 113: 265-275.

5. Elbouz M, Alfalou A, Brosseau C, Ben Haj Yahia N, Alam M S, 2015. Assessing the performance of a motion tracking system based on optical joint transform correlation. Opt. Commun. 349: 65-82.

6. Michael J, 2000. The influence of SLM pixel size and shape on the performance of optical correlators and optical memories. Proc. SPIE. 4089: 198-207.

7. Robert M Bunch, Jeffrey A Davis, Don M Cottrell, Ethan A Merrill, 2005. Effects of sampling and binarization in the output of the joint Fourier transform correlator. Opt. Eng. 29: 10941100. 
8. Joewono W, 2015. Noise-robust low-contrast retinal recognition using compression-based joint wavelet transform correlator. Opt. Las. Techol. 74: 97-102.

9. Isha M, Sudheesh K R and Naveen K N, 2014. Cryptanalysis of an image encryption scheme based on joint transform correlator with amplitude- and phase-truncation approach. Opt. Las. Eng. 52: 167-173.

10. Qian Yixian, Hong Xueting, Miao Hua, 2013. Improved target detection and recognition in complicated background with joint transform correlator. Optik. 124: 6282-6285.

11. Leonard I, Alfalou A, Alam MS, Arnold-Bos A, 2012 Adaptive nonlinear fringe-adjusted joint transform correlator. Opt. Eng. 51: 098201.

Chao Fan. 2017. Design of compact joint transform correlator. Ukr.J.Phys.Opt. 18: 55 - 65.

Анотація. Для поліпшення якості знімків просторових камер ми вимірюємо субпіксельні зсуви між двома сусідніми зображеннями, використовуючи корелятор спільного перетворення (КСП). Спочатку ми описуємо принципи вимірювання зсувів зображень на основі КСП. Далі з урахуванням дискретної піксельно-подібної будови ПЗС і просторового модулятора світла обговорено зв'язки між параметрами компонентів КСП, щзо випливають із теореми дискретизації та принциипів фур'є-оптики. На иій підставі обрано необхідні прилади і розраховано лінзу Фур'є для побудови компактного КСП. Нарешті, створено необхідну експериментальну базу і очінено ефективність вимірювань зсувів зображень. Результати засвідчили, щуо субпіксельні зсуви зображень можна виміряти з досить високою точністю. Похибки є меншими за 0,1 піксель за умови, щуо зсуви зображень перебувають у межах до 1 пікселя. 